\title{
Mutation of Arabidopsis SMC4 identifies condensin as a corepressor of pericentromeric transposons and conditionally expressed genes
}

\author{
Jing Wang, ${ }^{1,2}$ Todd Blevins, ${ }^{1,2,3,6}$ Ram Podicheti, ${ }^{4,5}$ Jeremy R. Haag, ${ }^{7}$ Ek Han Tan, ${ }^{8}$ Feng Wang, ${ }^{1,2}$ \\ and Craig S. Pikaard ${ }^{1,2,3}$ \\ ${ }^{1}$ Department of Biology, Indiana University, Bloomington, Indiana, 47405, USA; ${ }^{2}$ Department of Molecular and Cellular \\ Biochemistry, Indiana University, Bloomington, Indiana, 47405, USA; ${ }^{3}$ Howard Hughes Medical Institute, Indiana University, \\ Bloomington, Indiana, 47405, USA; ${ }^{4}$ Center for Genomics and Bioinformatics, Indiana University, Bloomington, Indiana, 47405 , \\ USA; ${ }^{5}$ School of Informatics and Computing, Indiana University, Bloomington, Indiana, 47405, USA
}

In eukaryotes, transcriptionally inactive loci are enriched within highly condensed heterochromatin. In plants, as in mammals, the DNA of heterochromatin is densely methylated and wrapped by histones displaying a characteristic subset of post-translational modifications. Growing evidence indicates that these chromatin modifications are not sufficient for silencing. Instead, they are prerequisites for further assembly of higher-order chromatin structures that are refractory to transcription but not fully understood. We show that silencing of transposons in the pericentromeric heterochromatin of Arabidopsis thaliana requires SMC4, a core subunit of condensins I and II, acting in conjunction with CG methylation by MET1 (DNA METHYLTRANSFERASE 1), CHG methylation by CMT3 (CHROMOMETHYLASE 3), the chromatin remodeler DDM1 (DECREASE IN DNA METHYLATION 1), and histone modifications, including histone H3 Lys 27 monomethylation (H3K27me1), imparted by ATXR5 and ATXR6. SMC4/condensin also acts within the mostly euchromatic chromosome arms to suppress conditionally expressed genes involved in flowering or DNA repair, including the DNA glycosylase ROS1, which facilitates DNA demethylation. Collectively, our genome-wide analyses implicate condensin in the suppression of hundreds of loci, acting in both DNA methylation-dependent and methylation-independent pathways.

[Keywords: DNA methylation; SMC proteins; chromosome condensation; epigenetic regulation; gene silencing; heterochromatin formation]

Supplemental material is available for this article.

Received May 5, 2017; revised version accepted August 7, 2017.

Condensins are multisubunit protein complexes named for their ability to catalyze ATP-dependent condensation of newly replicated chromosomes (Wood et al. 2010; Hirano 2016; Uhlmann 2016). Two condensin subtypes (I and II) have at their core a heterodimer of the STRUCTURAL MAINTENANCE OF CHROMOSOMES (SMC) ATPases SMC2 and SMC4, which are highly conserved and essential for viability. Three additional subunits, each having paralogs that differ in condensins I and II, interact with the SMC2-SMC4 heterodimers, forming pentameric

Present addresses: ${ }^{6}$ UPR 2357, Institut de Biologie Moléculaire des Plantes, Centre National de la Recherche Scientifique, Université de Strasbourg, F-67000 Strasbourg, France; ${ }^{7}$ Monsanto Company, Chesterfield, MO 63017, USA; ${ }^{8}$ School of Biology and Ecology, University of Maine, Orono, ME 04469, USA.

Corresponding author: cpikaard@indiana.edu

Article published online ahead of print. Article and publication date are online at http://www.genesdev.org/cgi/doi/10.1101/gad.301499.117. complexes that can topologically entrap DNA sequences brought together by looping, loop stacking, or other long-range interactions, thereby compacting the DNA. In addition to roles in mitosis, condensins affect genome organization and recombination and DNA repair, with a number of studies also implicating condensin in the repression of specific genes (Lupo et al. 2001; Bhalla et al. 2002; Dej et al. 2004; Machin et al. 2004; Meyer 2010; Wood et al. 2010; Rawlings et al. 2011; Jeppsson et al. 2014; He et al. 2016).

Much of what is known about condensin's effects on gene regulation stems from studies conducted using yeast,

(C) 2017 Wang et al. This article is distributed exclusively by Cold Spring Harbor Laboratory Press for the first six months after the full-issue publication date (see http://genesdev.cshlp.org/site/misc/terms.xhtml). After six months, it is available under a Creative Commons License (Attribution-NonCommercial 4.0 International), as described at http://creativecommons.org/licenses/by-nc/4.0/. 
Drosophila, or Caenorhabditis elegans, whose genomes lack appreciable DNA methylation. However, genomic cytosine methylation is common in eukaryotes, including plants and mammals (Law and Jacobsen 2010). The majority of cytosine methylation occurs at CG motifs and is accomplished by orthologous enzymes in mammals and plants; namely, the cytosine methyltransferases DNMT1 (DNA METHYLTRANSFERASE 1; mammals) or MET1 (plants). CG motifs are symmetrical in duplex DNA, and hemimethylated pairs of CG motifs are recognized by UHRF (mammals) or VIM (plants) proteins to facilitate DNMT1 or MET1 recruitment, thereby maintaining methylation on both strands (Bostick et al. 2007; Woo et al. 2007; Hashimoto et al. 2008). CHG methylation (where $\mathrm{H}$ is $\mathrm{A}, \mathrm{C}$, or $\mathrm{T}$ ) is also symmetric and can be maintained in Arabidopsis by CMT3 (CHROMOMETHYLASE 3). CMT3 has a chromodomain that binds histone H3 Lys9 (H3K9) dimethylated by SUVH4 (or related paralogs), and SUVH4 in turn binds methylated CHG, allowing CHG methylation and H3K9 methylation (H3K9me) to specify and maintain one another (Law and Jacobsen 2010).

Pericentromeric regions account for most of the constitutive heterochromatin in Arabidopsis (Fransz et al. 2002; Zhang et al. 2006). These regions are transposon-rich and gene-poor, with dense CG maintenance methylation required to keep the transposons inactive (Soppe et al. 2002; Lippman et al. 2004; Simon et al. 2015). However, transposons located elsewhere, particularly in the mostly euchromatic chromosome arms, require additional methylation by DRM2, the ortholog of mammalian DNMT3 enzymes. DRM2 methylates cytosines in CG, CHG, or $\mathrm{CHH}$ motifs in an RNA-directed manner (Cao and Jacobsen 2002; Cao et al. 2003; Zemach et al. 2013; Matzke and Mosher 2014; Wendte and Pikaard 2017). In some contexts, primarily pericentromeric heterochromatin, $\mathrm{CHH}$ methylation can be maintained by the DNA methyltransferase CMT2 (Zemach et al. 2013; Stroud et al. 2014). CG, $\mathrm{CHG}$, and $\mathrm{CHH}$ maintenance methylation has at least one thing in common; namely, the need for the chromatin remodeling ATPase DDM1 (DECREASE IN DNA METHYLATION 1) (Jeddeloh et al. 1999; Brzeski and Jerzmanowski 2003), which enables maintenance methylation within regions of dense heterochromatin enriched for linker histone $\mathrm{Hl}$ (Zemach et al. 2013).

Here, we report a hitherto unrecognized role for condensins I and II in methylation-dependent repression of pericentromeric transposons whose silencing depends on MET1, CMT3, DDM1, and the H3K27 monomethylases ATXR5 and ATXR6 (Jacob et al. 2009, 2010). Cytosine methylation is not appreciably altered in smc4 mutants, suggesting that condensin is not required for DNA methylation but acts in conjunction with DNA methylation to assemble higher-order repressive chromatin complexes. We also show that SMC4/condensin does not act solely at heavily methylated loci of pericentromeric regions but also represses sparsely methylated, conditionally expressed genes throughout the chromosome arms, suggesting a broad role in shaping the Arabidopsis epigenome.

\section{Results}

Overexpression of the NRPE1 C-terminal domain (CTD) results in defective RNA-directed DNA methylation (RdDM)

Our finding that SMC4/condensin regulates gene silencing came about unexpectedly through studies of RdDM, which involves two specialized multisubunit RNA polymerases: polymerase IV (Pol IV) and Pol V (Haag and Pikaard 2011; Matzke and Mosher 2014; Zhou and Law 2015; Wendte and Pikaard 2017). We found that overexpressing the CTD of the Pol V largest subunit, NRPE1, causes a dominant-negative phenotype resembling nrpe1 loss-of-function mutants (Fig. 1). For instance, at 45S and 5S rRNA gene loci, Pol IV-dependent 24-nucleotide (nt) siRNAs that are diminished in a nrpe1 mutant are similarly reduced in the NRPE1 CTD overexpression line CTD-OX (Fig. 1A). Likewise, RdDM at AtSN1 and SoloLTR retrotransposons is lost in the nrpe1-11 mutant and greatly reduced in CTD-OX plants, making the DNA of these elements susceptible to HaeIII or AluI digestion such that PCR amplification fails (Fig. 1B).

Knocking out both DRM2-mediated RdDM and CHG methylation by CMT3 causes overexpression of the Fbox gene $S D C$, resulting in plants with elongated twisted leaves (Henderson and Jacobsen 2008). We found that cmt3 CTD-OX double mutants, like drm1 drm2 cmt3 mutants, display strong SDC expression (Fig. 1C) and the characteristic twisted leaf phenotype (Fig. 1D). AtSN1 and soloLTR retrotransposons are also highly expressed in CTD-OX plants, as in nrpe1 mutants (Fig. 1C). We assayed endogenous NRPE1 mRNA expression levels using both RT-PCR and quantitative RT-PCR (qRT-PCR) assays, detecting an increase in the nrpd1-3 (pol IV) mutant but no change in NRPE1 mRNA levels in CTD-OX plants (Fig. 1E; Supplemental Fig. S1A), suggesting CTD-OX interference with RdDM at a step downstream from NRPE1 transcription.

\section{Evidence that the CTD-OX transgene induces RNAi}

Using a homozygous cmt3 CTD-OX line in which all progeny displayed the SDC phenotype, we conducted a suppressor screen. Seeds were subjected to EMS mutagenesis, and rare plants with a wild-type phenotype were identified in the next generation (M2). These plants no longer expressed $S D C$, as illustrated in Figure $1 \mathrm{~F}$ for four such mutants (m17, m65, m71, and $\mathrm{m} 73)$. In the mutants, AtSN1 and soloLTR elements that had been derepressed in the cmt3 CTD-OX parental line (a pol $\mathrm{V}$ or RdDM mutant phenotype), were resilenced (Fig. 1F) and remethylated (Fig. 1G), and the CTD transgene remained expressed (Fig. 1F; Supplemental Fig. S1B), ruling out its silencing as the basis for the suppressor phenotype.

Evidence that recovered mutants were affecting more than one activity came from analyses of small RNAs. These included 24-nt siRNAs matching $5 S$ rRNA genes or AtSN1 elements; 21-nt secondary siRNAs generated from TAS1, TAS2, or TAS3 noncoding RNAs (Allen et al. 2005); or a 21-nt microRNA (miR160) (Fig. 2A). In 


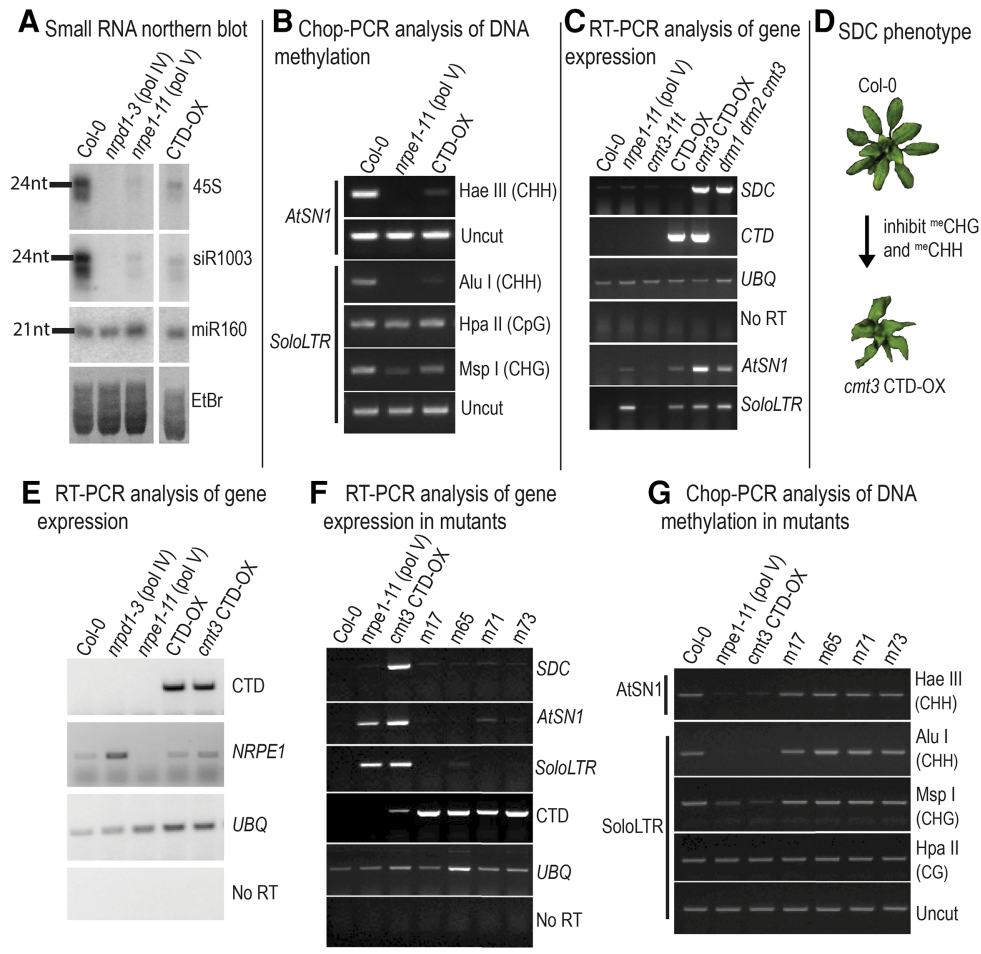

Figure 1. Overexpression of the Pol V largest subunit CTD induces a dominant-negative RdDM phenotype suppressed in EMS-induced mutants. (A) RNA blot analysis of small RNA of wild-type (Col-0), pol IV mutant (nrpd1), pol V mutant (nrpe1-11), or CTD-OX plants. The blot was sequentially probed for small RNAs matching the $45 S$ rRNA gene promoter, $5 S$ rRNA gene intergenic spacer (siR1003), or microRNA miR160. An image of the ethidium bromide (EtBr)-stained gel is shown at the bottom. (B) Analysis of AtSN1 and SoloLTR transposon DNA methylation levels using Chop-PCR. Genomic DNA of wild-type Col-0, Pol V mutant (nrpe1-11), or CTD-OX plants was digested (chopped) with the indicated methylation-sensitive endonucleases (the sequence context of queried cytosines are shown in parentheses) or left uncut as a control and then amplified using PCR primers specific for AtSN1 or soloLTR retrotransposons. PCR products were resolved by agarose gel electrophoresis and visualized with $\mathrm{EtBr}$ staining. (C) RT-PCR analyses of SDC, CTD, AtSN1, and soloLTR expression levels relative to a ubiquitin $(U B Q)$ control. The genotypes of plants tested are indicated at the top of each lane. Reactions in which reverse transcriptase was omitted (no RT) control for DNA contamination. The $d r m 1 \mathrm{drm} 2 \mathrm{cmt} 3$ genotype is known to induce $S D C$ overexpression, serving as a positive control for the cmt3 CTD-OX genotype. The CTD reactions control for transgene expression. (D) CHG and $\mathrm{CHH}$ methylation-deficient $\mathrm{cmt} 3 \mathrm{CTD}-\mathrm{OX}$ plants display the SDC overexpression phenotype. (E) RT-PCR analysis of CTD and native NRPE1 expression. The genotypes of plants tested are indicated at the top of each lane. Reactions lacking reverse transcriptase (no RT) control for DNA contamination. UBQ reactions control for the amount of RNA tested. (F) RT-PCR analysis of SDC, AtSN1, soloLTR, and CTD expression in the cmt3 CTD-OX parental line and in the suppressor mutants $\mathrm{m} 17, \mathrm{~m} 65, \mathrm{~m} 71$, and $\mathrm{m} 73$. The nrpe1-11 (pol V) mutant served as control for derepression of AtSN1 and SoloLTR elements silenced by RdDM in wild type (Col-0). UBQ served as a loading control. Reactions without reverse transcriptase (no RT) served as controls for DNA contamination. (G) Analysis of AtSN1 and SoloLTR DNA methylation levels using Chop-PCR. Assays were conducted as in $B$, comparing wild-type Col-0 with the indicated mutants.

all four mutants, 24-nt siRNAs increased from pol $\mathrm{V}$ (nrpe1) mutant levels to wild-type levels, but, in $\mathrm{m} 17$, TAS locus siRNAs (tasiRNAs) were absent. Production of tasiRNAs requires RNA-DEPENDENT RNA POLYMERASE 6 (RDR6) to generate the double-stranded precursors that are then diced into 21-nt RNAs (Peragine et al. 2004; Vazquez et al. 2004; Allen et al. 2005). This prompted genetic tests that revealed that mutant $\mathrm{m} 17$ is not complemented upon crossing to an $r d r 6$ mutant (see Supplemental Fig. S2A,B). Subsequent sequencing of the $R D R 6$ gene in $\mathrm{m} 17$ revealed a G-to-A transition, changing Gly866 to a glutamate at the enzyme's active site (Fig. 2B), the same mutation as in previously identified rdr6-13 and sgs2-6 alleles (Mourrain et al. 2000; Peragine et al. 2004). Additional genetic tests revealed that mutation of SGS3, which enables RDR6 function (Peragine et al. 2004; Vazquez et al. 2004), also suppresses SDC, AtSN1, and soloLTR expression in $c m t 3 C T D-O X$ plants (Supplemental Fig. S2C), as does mutation of ARGONAUTE 1 (AGO1) (Supplemental Fig. S2D), which binds 21-nt siRNAs (Mallory and Vaucheret 2010).

Although NRPE1 expression levels are not appreciably affected by the CTD-OX transgene (see Fig. 1E; Supplemental Fig. S1), the fact that $r d r 6$, sgs3, and ago1 mutations prevent SDC, AtSN1, and soloLTR overexpression in cmt3 CTD-OX plants strongly implicated 21-nt siRNA-mediated RNAi as the basis for CTD-OX action. This led us to examine whether 21-nt siRNAs matching the NRPE1 CTD are produced in cmt3 CTD-OX plants but lost in the mutants. Indeed, this is the case, as shown in Figure 2C.

\section{A missense allele of the condensin subunit gene SMC4 suppresses the SDC phenotype}

Unlike the $r d r 6$ mutant (m17), mutant m73 showed no loss of 21-nt tasiRNAs (Fig. 2A) yet had lost 21-nt siRNAs matching the NRPE1 CTD (Fig. 2C), suggesting a defect in transgene-induced RNAi but not tasiRNA biogenesis. The recessive nature of the $\mathrm{m} 73$ mutation and the dominant nature of the CTD-OX transgene for 21-nt siRNA production were evident upon crossing $\mathrm{m} 73$ with wildtype Col-0, yielding F1 progeny expressing high levels of 21-nt siRNAs matching the NRPE1 CTD (Fig. 2C).

Using bulked-segregant analysis coupled with deep genome sequencing, the causative mutation in m73 was identified as a C-to-T transition in the SMC4 gene (AT5G48600), defining the allele smc4-1. The missense mutation in smc4-1 results in a proline-to-serine substitution at amino acid 22 (P22S) at the edge of the conserved 


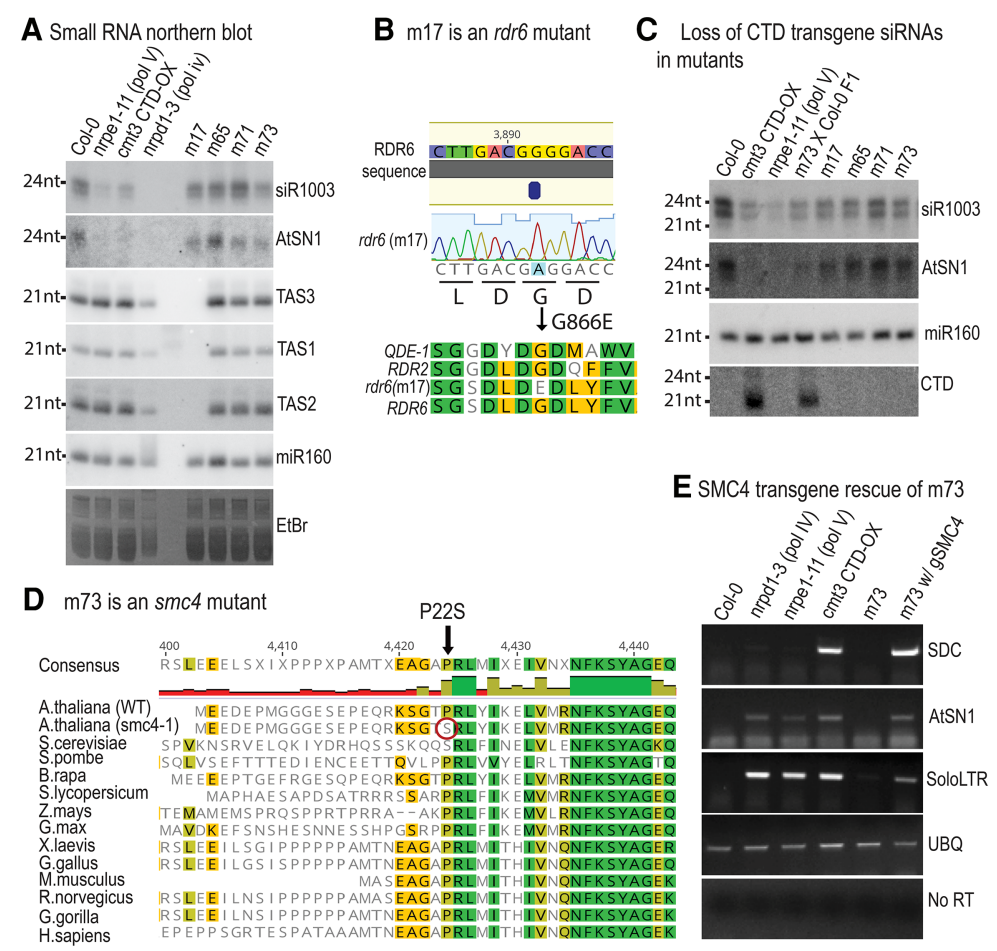

Figure 2. Suppressors of the SDC phenotype of $\mathrm{cmt} 3$ $C T D-O X$ plants include mutant alleles for RDR6 and the condensin core subunit SMC4. (A) Small RNA blot analyses. RNA isolated from the indicated genotypes was resolved by polyacrylamide gel electrophoresis and blotted to membranes that were then sequentially probed using body-labeled RNA oligonucleotides corresponding to the $5 S$ rRNA gene intergenic spacer (siR1003), TAS3, TAS1, TAS2, miR160, or AtSN1. The nrpd1-3 (pol IV) and nrpe1-11 (pol V) mutants were positive controls for loss or reduction, respectively, of 24-nt siR1003 and AtSN1 siRNAs. An image of the EtBrstained gel is shown in the bottom panel. (B) Suppressor mutant $\mathrm{m} 17$ is an $r d r 6$ mutant. A guanosine-to-adenosine transition in the RDR6 gene causes an amino acid change, G866E, in the enzyme active site. The multiple alignment compares active site regions of wild-type RDR6, m17 RDR6, Neurospora QDE-1, and Arabidopsis RDR2. $(C)$ The $C T D$-OX transgene generates 21-nt CTD siRNAs that are lost in suppressor mutants. RNA blots for the indicated genotypes were sequentially probed for siR1003 or AtSN1 24-nt siRNAs, miR160, or CTD small RNAs. (D) Mutant $\mathrm{m} 73$ harbors the mutant allele smc41. Multiple sequence alignment of the SMC4 N-terminal region in diverse eukaryotes highlighting the P22S mutation present in suppressor mutant $\mathrm{m} 73$, defining the smc4-1 allele. Identical amino acids are highlighted in green, and similar amino acids are highlighted in yellow. (E) SMC4 transgene rescue of the smc4-1 mutant. RT-PCR analyses of SDC, AtSN1, SoloLTR, and UBQ (control) expression in the indicated genotypes, including the $\mathrm{cm} t 3$ CTD-OX parental line used to conduct the suppressor screen, the $\mathrm{m} 73(\mathrm{smc4}-1) \mathrm{mutant}$, and $\mathrm{m} 73$ transformed with an SMC4 transgene ( $g S M C 4)$ that reverts the expression pattern to that of the starting $c m t 3 C T D$-OX line.

ATPase domain (Fig. 2D). Because a T-DNA insertion allele of smc4 is embryonic-lethal when homozygous (Siddiqui et al. 2006; Smith et al. 2014), smc4-1 is likely a hypomorphic allele.

To confirm that the smc4-1 mutation is causative, we transformed the $\mathrm{m} 73$ mutant with a transgene expressing a full-length $S M C 4$ gene ( $g S M C 4)$, which restored $S D C$ expression (Fig. 2E). Likewise, AtSN1 and soloLTR elements that had been silenced in the smc4-1 (m73) mutant were again derepressed as in the starting cmt3 CTD-OX line (Fig. 2E) or in nrpd1 (pol IV) or nrpe1 (pol V) mutants.

Collectively, the results of the mutant screen are best explained by the interpretation that aberrant RNAs generated by the single-copy $C T D$-OX transgene give rise to RDR6- and SGS3-dependent 21-nt siRNAs that disrupt Pol V activity via RNAi. This impairs RdDM, resulting in SDC, AtSN1, and soloLTR expression. In the RNAi mutants, Pol V activity is no longer disrupted. We deduce that SMC4, as a key subunit of condensin, is somehow required for the CTD-OX transgene to produce aberrant RNAs.

To circumvent the uncertainties associated with the cmt3 CTD-OX genetic background, we outcrossed m73 to wild-type Col-0 and identified F2 progeny that were homozygous for smc4-1, homozygous wild-type for CMT3, and devoid of the CTD-OX transgene. These smc4-1 homozygotes were further backcrossed twice to wild-type Col-0, yielding a homozygous line referred to here as smc4-1 (Col-0) or simply smc4-1. This line was used for all subsequent assays.
SMC4 is required for pericentromeric transposon silencing and chromocenter condensation

Genome-wide effects of SMC4 on gene expression were investigated by conducting mRNA deep sequencing comparing smc4-1 with wild-type plants. The most striking finding was that hundreds of transposable elements (TEs) are derepressed to high levels in smc4-1 (Fig. 3A,B; Supplemental Table S1), most by a factor of $\sim 16$-fold to 100-fold compared with wild-type (Fig. 3B, note the $\log _{2}$ scale for the $Y$-axis). Nearly $80 \%$ are retrotransposons belonging to three superfamilies (LTR/gypsy, LTR/Copia, and Line/L1) (Fig. 3A), but En/Spm DNA transposons also represent a substantial subset. The derepressed TEs map primarily to the centromeric and pericentromeric regions of all five chromosomes (Fig. 3B) and tend to be long TEs, as categorized by Zemach et al. (2013) (Fig. 3C).

Using RT-PCR, we confirmed the derepression in smc4-1 of several TEs identified by RNA sequencing (RNA-seq), comparing these with soloLTR, which is silenced by RdDM, while also testing a variety of mutants, including the RdDM mutants nrpd1 (pol IV), nrpe1 (pol $V)$, and drm1 drm2 or the maintenance methylation mutants met1, cmt2, and cmt3 (Fig. 3D). Strikingly, TEs derepressed in smc4-1 are also derepressed in met 1 or cmt3 mutants but not in RdDM or cmt2 mutants (Fig. 3D). Collectively, these results suggest that condensin is needed in addition to CG and CHG methylation for silencing of TEs located in pericentromeric heterochromatin. Consistent with this interpretation, 
A Derepressed TEs classified by superfamily

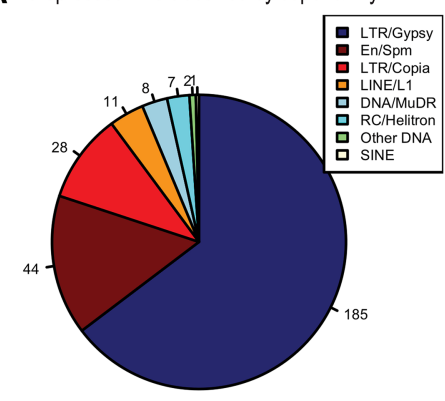

C Size distribution of derepressed TEs

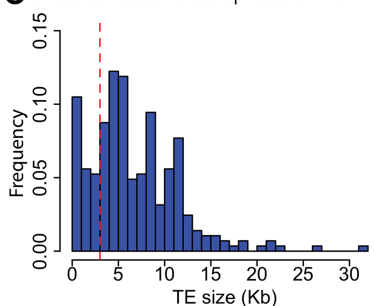

E Chromocenter condensation status

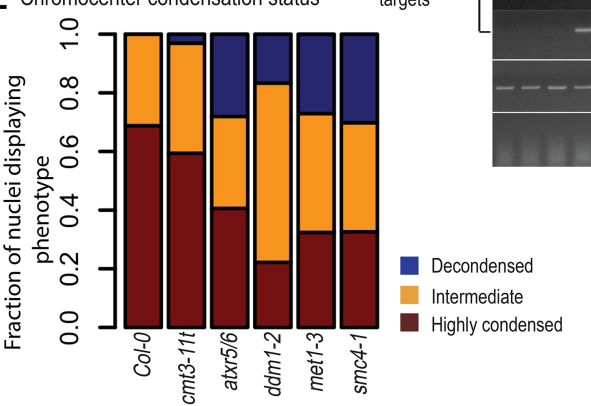

B Chromosomal positions of derepressed TEs

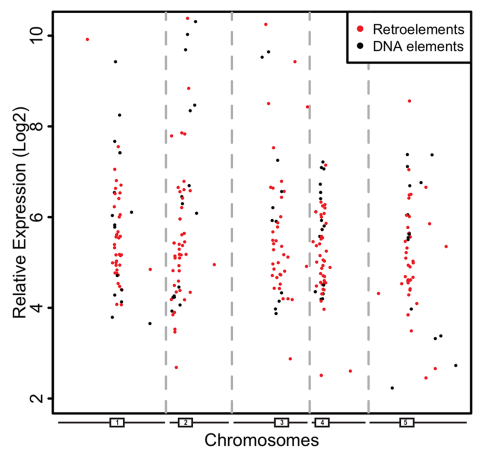

D RT-PCR verification of TE expression
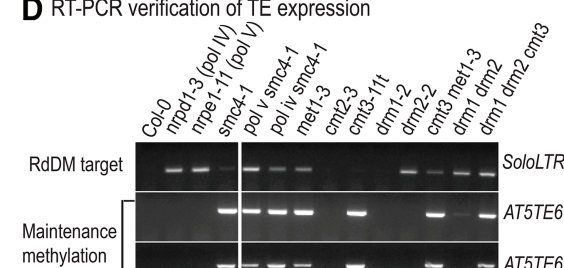

et

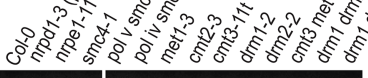

\section{oLTR}

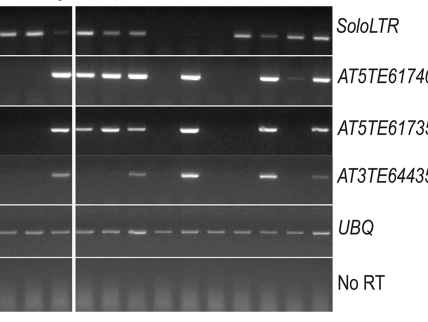
of RdDM) assayed by RT-PCR in mutants representing the RdDM pathway (nrpd1, nrpe1, drm1-2, and drm22), CG or CHG maintenance methylation pathways (met1-3, cmt2-3, and cmt3-11t), or the CHH maintenance methylation pathway (cmt2-3). UBQ and reactions from which reverse transcriptase was omitted (no RT) served as controls. (E) Relative frequencies of decondensed, partially decondensed (intermediate), or wildtype chromocenters in DAPI-stained nuclei of cmt311t, atxr5/6, ddm1-2, met1-3, smc4-1, or wild-type Col-0.

Figure 3. TEs in pericentromeric regions are derepressed in smc4-1 mutants. (A) TEs derepressed at least with a false discovery ret/wild-type value of $\log _{2}>2$, are classified by superfamily. (B) Chromosomal positions of TEs derepressed fourfold or more in smc4-1. Retrotransposons are denoted by red dots, and DNA mere postions are by black dots. Estimated centroleft to right), whose centromeres are represented by rectangles. (C) Size distribution of the 286 SMC4-repressed TEs. The $X$-axis shows the annotated TE size in kilobases, and the $Y$-axis shows the relative frequency of ele$\mathrm{kb}$, beyond which TEs are classified as "long." $(D)$ Overlap between TEs repressed by SMC4 and TEs repressed by maintenance cytosine methylation. Expression of three TEs derepressed in smc4-1 (AT5TE61740,

heterochromatic chromocenters become decondensed in smc4-1 mutants as in met 1, cmt3, or ddm 1 mutants (Fig. 3E; Supplemental Fig. S3; see also Soppe et al. 2002).

MET1, DDM1, H3K9 dimethylation (H3K9me2), H3K27 monomethylation (H3K27me1), and SMC4 silence overlapping subsets of TES

To examine how transposons silenced by SMC4 overlap with transposons silenced by CG or CHG methylation, we compared smc4-1 mRNA-seq data with published (Stroud et al. 2013) met1, ddm1, cmt3, or suvh4/5/6 mRNA-seq data sets (Fig. 4). Two-thirds of all TEs derepressed fourfold or more in met 1 are also derepressed in $d d m 1$ mutants (636 of 956) (Fig. 4A), as reported previously (Stroud et al. 2013). Fewer TEs are derepressed in smc41 (286) than in met1 (956), but $63 \%$ (181 of 286) of these SMC4-dependent TEs overlap with TEs derepressed in met 1 , which is highly significant $\left(P=3.60 \times 10^{-203}\right)$ given an expectation of only $3.1 \%$ overlap by chance. It is important to note that smc4-1 mutants are viable, whereas a smc4 T-DNA insertion is lethal. Thus, the number of TEs up-regulated in smc4-1 as a loss-of-function allele but not a null allele may underestimate the full effects of SMC4. Fifty-nine percent (169 of 286) of the TEs dere- pressed in smc4-1 are derepressed in both met 1 and $d d m 1$ (Fig. 4A), which is also highly significant ( $P$ value of essentially 0 ) given an expected overlap (by chance) of only $0.08 \%$.

In cmt3-11 mutants, only 32 TEs are derepressed fourfold or more; however, $47 \%$ (15 of 32 ) of these overlap with TEs derepressed in smc4-1 (Fig. 4B)-significantly more than the $0.92 \%$ expected by chance $(P=9.27 \times$ $\left.10^{-23}\right)$. Moreover, $31 \%$ (10 of 32) of the TEs derepressed in cmt3 overlap with TEs derepressed in smc4-1, met1, and $d d m 1$ (Fig. 4B,C)-significantly more than the $0.0007 \%$ expected by chance $\left(P=1.99 \times 10^{-44}\right)$.

Comparing smc4-1 data with published nrpe1 (pol V) mRNA-seq data (Blevins et al. 2014), only one derepressed TE was common to both data sets (Supplemental Fig. S4A), which is not statistically significant $(P=0.25)$. TEs derepressed in smc4-1 also showed no further increase in expression in smc4-1 nrpe 1 or smc4-1 nrpd1 double mutants (Supplemental Fig. S4B). Collectively, the results implicate condensin in MET1-, CMT3-, and DDM1-dependent transposon silencing but not silencing by RdDM.

Turning from DNA to histone methylation, 139 TEs are derepressed in a triple mutant for the H3K9 dimethylases SUVH4, SUVH5, and SUVH6. Of these TEs, $26.6 \%$ (37 of 139) are also derepressed in smc4-1 (Fig. 4D), a 
A Derepressed TEs in smc4, met1 and ddm1

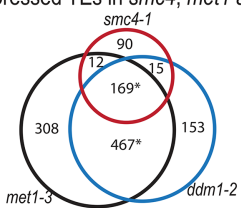

B

Derepressed TEs in smc4, met1 and $c m t 3$

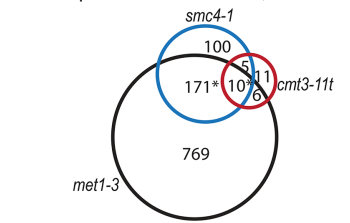

C

Derepressed TEs in smc4, ddm1 and cmt3

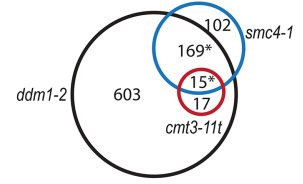

D Overlap between TEs derepressed in smc4-1 and suvh 4/5/6.

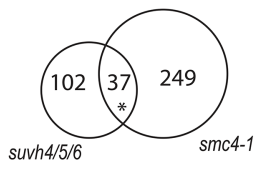

E

Comparison of TEs derepressed in atx $55 / 6$ to SMC4, MET1 and DDM1-dependent TEs

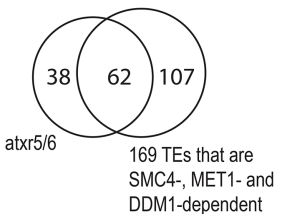

$\mathbf{F}$

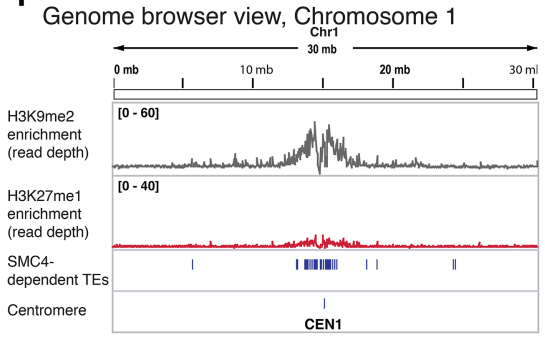

G ATXR5 and 6 co-repress SMC4, MET1 and DDM1 targets

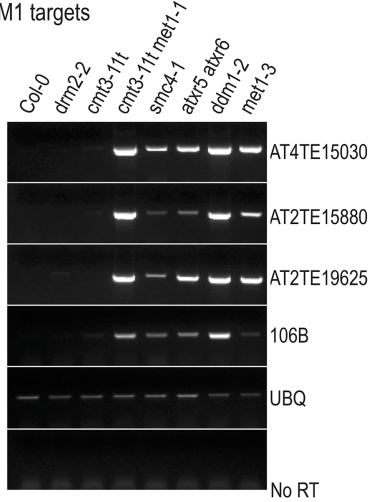

H Expression levels for the 169 TEs derepressed in smc4, met1 and ddm1 RNA-seq
read count

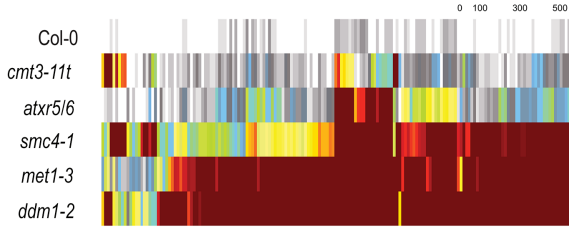

Figure 4. SMC4 coregulates loci silenced by maintenance DNA methylation and histone modifications associated with heterochromatin. (A) SMC4 silences a significant subset of MET1 and DDM1 targets. Venn diagram describing the relationship between TEs derepressed in the smc4-1, met1-3, and ddm1-2 mutants. Asterisks denote statistically significant overlaps. (B) Venn diagram showing the overlap between TEs derepressed in the smc4-1, met1-3, and cmt3-11t mutants. Asterisks denote statistically significant overlaps. $(C)$ Venn diagram describing the relationships between TEs derepressed in the smc4-1, ddm1-2, and cmt3-11t mutants. Asterisks denote statistically significant overlaps. (D) Overlap between TEs derepressed in smc4-1 and suvh4 suvh5 suvh6 triple mutants. Asterisks denote statistically significant overlaps. (E) ATXR5 and ATXR6 help silence a subset of TEs that also require MET1, DDM1, and SMC4. The Venn diagram compares the 100 TEs derepressed in an atxr5 atxr6 double mutant with the 169 TEs that represent the overlap between the set of TEs derepressed in met1, $d d m 1$, and smc4-1. (F) Genome browser snapshot of H3K9me2 enrichment, H3K27me1 enrichment, localization of SMC4-dependent TEs, and the centromeric position on chromosome 1 in wild-type Col0 . Raw counts of fragment pileup for the ChIP-seq (chromatin immunoprecipitation [ChIP] combined with high-throughput sequencing) data are shown in the top two tracks. Vertical bars in the third and fourth tracks represent SMC4-dependent TEs or centromeric repeats, respectively. (G) RT-PCR verification of the derepression of four TEs (AT4TE15030, AT2TE15880, AT2TE19625, and 106B) predicted from RNA-seq data to be silenced via the partnership of MET1, DDM1, ATXR5/6, and SMC4. Genotypes tested are indicated at the top of the figure. UBQ reactions served as loading controls. Reactions omitting reverse transcriptase (no RT) control for DNA contamination. $(H)$ Hierarchical clustering of the 169 TEs coregulated by SMC4, MET1, and DDM1, with TE expression levels displayed as a heat map. Expression levels were determined as RNA-seq reads corresponding to the TEs, normalized to the total number of mapped reads per genotype.

significant fraction $\left(P=1.2 \times 10^{-43}\right)$, indicating that a subset of TEs requires both $\mathrm{H} 3 \mathrm{~K} 9 \mathrm{me} 2$ and SMC4 for silencing. Using the H3K9me2 ChIP-seq (chromatin immunoprecipitation [ChIP] combined with high-throughput sequencing) data of Stroud et al. (2014), we plotted the density of $\mathrm{H} 3 \mathrm{~K} 9 \mathrm{me} 2$ (relative to total H3) at the 286 TEs derepressed in smc4-1, comparing these elements with a training set of 313 TEs randomly selected from the Arabidopsis thaliana genome (see Supplemental Tables S5, S6 for the lists of transposons examined). SMC4-dependent TEs show H3K9me2 enrichment throughout the elements in wild-type plants (Supplemental Fig. S5A) and lose this enrichment in suvh4 suvh5 suvh6 triple mutants (Supplemental Fig. S5B). In contrast, the 313 randomly selected TEs show a lesser degree of H3K9me2 enrichment that is independent of SUVH4/5/ 6. Interestingly, genes whose repression involves SMC4 show no enrichment for H3K9me2 (Supplemental Fig. $\mathrm{S} 5 \mathrm{~A}, \mathrm{~B})$, indicating that not all SMC4 targets are enriched for $\mathrm{H} 3 \mathrm{~K} 9 \mathrm{me} 2$.
The H3K27 monomethylases ATXR5 and ATXR6 are functionally redundant paralogs important for the stability and silencing of pericentromeric heterochromatin (Jacob et al. 2009, 2010). TEs derepressed in atxr5/6 double mutants show substantial overlap with TEs derepressed in $d d m 1$ or met1 (Stroud et al. 2012). The H3K27me1 mark is enriched within the bodies of SMC4-dependent TEs as well as randomly selected TEs but not genes (Supplemental Fig. S5C). Therefore, we compared TEs derepressed fourfold or more in atxr5/6 with the 169 TEs whose silencing is SMC4-, MET1-, and DDM1-dependent, revealing a $37 \%$ (62 of 169) overlap (Fig. 4E), which is significantly higher than the $0.32 \%$ expected by chance $(P=$ $\left.4.34 \times 10^{-119}\right)$.

Collectively, the overlap between H3K9me2, H3K27me1, and TEs repressed by SMC4 correlates with the enrichment of all three features in pericentromeric regions, as shown for chromosome 1 in Figure 4F.

As a test of the RNA-seq results, we examined the expression status of four TEs whose silencing is dependent 
on MET1, DDM1, SMC4, and ATXR5/6 using RT-PCR assays (Fig. 4G). This gel-based assay confirmed the conclusions from the RNA-seq data, showing that the TEs are silenced in wild-type plants (Col-0) but derepressed in each of the mutants.

Displaying RNA-seq data as a heat map, we compared the relative expression levels of the 169 SMC4-, MET1-, and DDM1-dependent TEs in cmt3, atxr5/6, smc4, met1, or ddm1 mutants (Fig. 4H). DDM1 and MET1 exert the strongest repression of the largest number of TEs, with SMC4 also needed for moderate to strong repression in most cases. ATXR 5 and ATXR6 exert a less pronounced effect on TE expression, but a subset of TEs requires SMC4, MET1, DDM1, and ATXR5/6 for strong repression.

\section{DNA methylation and SiRNA accumulation are unaltered in smc4-1 mutants}

Because SMC4/condensin partners with cytosine methylation proteins in TE repression, we conducted genomewide bisulfite sequencing to test whether SMC4 is required for DNA methylation. Transposons that are derepressed in smc4-1 mutants are heavily methylated in all sequence contexts, as shown in the heat maps of Figure 5A. Their CG methylation is almost completely lost in met1 mutants, their CHG methylation is greatly diminished in cmt3 mutants, and their $\mathrm{CHH}$ methylation is depleted in cmt2 mutants (Fig. 5A). Methylation in all three of these sequence contexts is also substantially reduced in $d d m 1$ mutants but not in drm1 drm2 or nrpe1 (pol V) mutants, consistent with maintenance methylation rather than RdDM. Importantly, DNA methylation is not appreciably affected in any sequence context in smc4-1 mutants either at TEs regulated by SMC4 (Fig. 5A) or genome-wide (Supplemental Fig. S6A). This is also apparent upon examining methylation patterns at individual loci, as shown for three TE loci in Figure 5B.

Recall that transgene-induced 21-nt siRNAs matching the NRPE1 CTD region in the cmt3 CTD-OX line were absent in the m73 mutant. This led us to conduct small RNA deep sequencing (small RNA-seq) to see whether small RNA levels are affected by smc4-1 genome-wide. We detected no change in siRNA levels relative to wildtype Col-0 in the smc4-1 mutant for either 21- or 24-nt siRNAs (Fig. 5C; Supplemental Fig. S6B,C). This suggests that condensin somehow affects the CTD-OX transgene in a locus-specific manner without having genome-wide effects on 21-nt siRNA biogenesis.

MORC ATPases are thought to function downstream from DNA methylation to affect heterochromatin
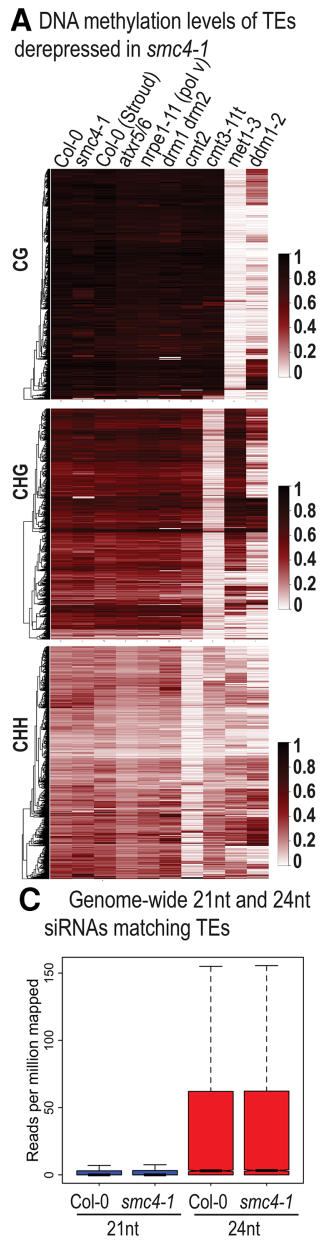

B RNA-seq and methylation profiles for three SMC4-dependent TES

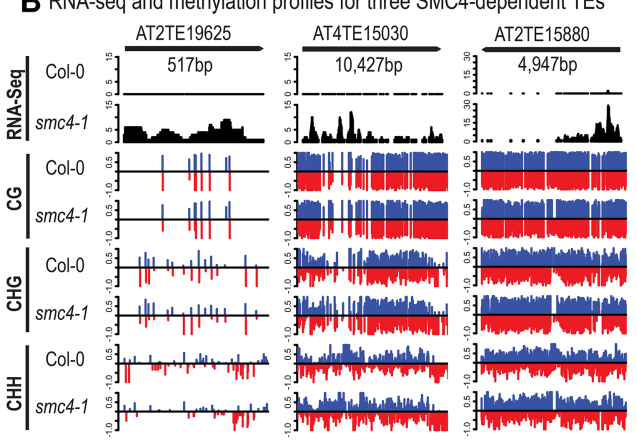

D TE derepression in condensin I or II mutants

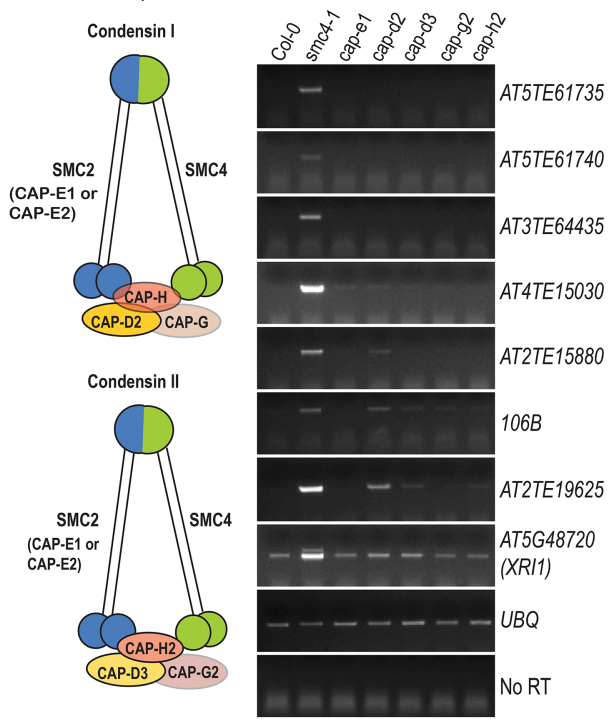

Figure 5. SMC4 does not affect cytosine methylation or siRNA levels and acts in the context of both condensins I and II. (A) Hierarchical clustering of the 286 SMC4dependent TEs displaying DNA methylation levels on a scale of 0.0 (white) to 1.0 (black) in smc4-1, atxr5/6, nrpe1 (pol V), drm1 drm2, cmt2, cmt3, met1, and $d d m 1$ mutants. Col-0 was the control from our study, and Col-0 (Stroud) was the control for the mutant methylome data of Stroud et al. (2012). Columns represent data for each indicated genotype, and rows represent 200-base-pair (bp) windows covering the 286 TEs. The rows were clustered by complete agglomeration hierarchical clustering method, with Euclidean distance as a distance measure. (B) RNA-seq and methylation profiles for three TEs (AT2TE19625, AT4TE15030, and AT2TE15880) cooperatively regulated by SMC4, MET1, DDM1, and ATXR5/6. The first two data tracks show mapped RNA-seq reads (black vertical bars) in the wild-type Col-0 and smc4-1 mutant. The remaining six data tracks show cytosine methylation levels in each of the three sequence contexts (CG, $\mathrm{CHG}$, and $\mathrm{CHH}$ ). Methylation on the plus strand is plotted with blue vertical bars, and methylation on the minus strand is plotted with red vertical bars. TEs (black bars) are shown above the data tracks. $(C)$ Box plot analyses comparing wild-type Col-0 and smc4-1 with respect to 21- and 24nt small RNA abundance genome-wide. All read counts were normalized to total mapped reads. $(D)$ Involvement of condensins I and II in SMC4-dependent silencing. The cartoons at the left show the subunit compositions of $A$. thaliana condensins I and II. The gel images show RTPCR results for SMC4-dependent loci, tested in the indicated condensin subunit mutants. 
condensation (Jacob and Martienssen 2012; Lorkovic et al. 2012; Moissiard et al. 2012), possibly in ways similar to SMC ATPases (Iyer et al. 2008). Analyzing mRNA-seq data for atmorc1 atmorc6 double mutants (Moissiard et al. 2012; Stroud et al. 2013), we found that 59\% (26 of 44) of the TEs derepressed in atmorc1/6 overlap with TEs derepressed in met1 and $d d m 1$ (Supplemental Fig. S7A), which is significantly higher than the $0.08 \%$ expected by chance $\left(P=1.07 \times 10^{-69}\right)$ and comparable with the $59 \%$ overlap between TEs derepressed in smc4-1, met1, and $d d \mathrm{~m}$. Of the TEs derepressed in atmorc1/6, 41\% (18 of 44) overlap with TEs derepressed in smc4-1 (Supplemental Fig. S7B).

Collectively, our results indicate that TEs silenced in an SMC4-dependent manner are subject to multiple levels of control, including cytosine hypermethylation, histone $\mathrm{H} 3 \mathrm{~K} 9 \mathrm{me}$ and $\mathrm{H} 3 \mathrm{~K} 27 \mathrm{me}$, and assembly into higher-order complexes that also involve MORC ATPases.

\section{Both condensins I and II are involved in SMC4- dependent transposon silencing}

SMC4 is a core subunit of condensin I and condensin II, making it unclear whether defective TE silencing in smc4-1 stems from impairment of one or both forms of condensin. To address this question, we identified and tested homozygous T-DNA insertion mutants disrupting additional condensin subunits (Fig. 5D). These included CAP-E1, which is one of two SMC2 paralogs potentially present in either (or both) forms of condensin; CAP-D2, which is specific for condensin I; and the condensin II-specific subunits CAP-D3, CAP-G2, and CAP-H2. RT-PCR assays for expression of seven SMC4-dependent TEs demonstrated that all are derepressed to the greatest extent in smc4-1 (Fig. 5D). In three cases (AT2TE15880, 106B, and AT2TE19625), condensin I appears to play the largest role, based on the level of TE expression in the cap-d2 mutant, but condensin II subunit mutants also display some degree of TE derepression, indicating that both condensins I and II are important for silencing these elements. At the other four TEs, single mutants affecting condensin I- or II-specific subunits have little effect, suggesting that condensins I and II are redundant for silencing such that only mutations affecting both (smc4-1) bring about their derepression. No significant TE derepression was observed for the cap-e1 mutant, suggesting that the CAPE1 and CAP-E2 paralogs may be functionally redundant forms of SMC2.

\section{SMC4/condensin represses sparsely methylated protein- coding genes}

In addition to derepression of TEs, 533 protein-coding genes are significantly up-regulated in smc4-1 mutants $(P<0.01$; false discovery rate $[\mathrm{FDR}]<0.05)$ (Supplemental Table S2). Unlike the TEs, which are mostly pericentromeric, the derepressed protein-coding genes are distributed throughout the chromosome arms (Fig. 6A). Also unlike the TEs, protein-coding genes regulated by SMC4 are only sparsely methylated (Fig. 6B).
Interestingly, 500 of the 533 up-regulated protein genes affect three major processes; namely, flower development, reproductive processes, and DNA repair (Fig. 6C; Supplemental Table S3). Up-regulation of four of the identified DNA repair genes (GMI1, BRCA1, XRI1, and RAD51) was verified by RT-PCR (Fig. 6D), consistent with a prior study that showed up-regulation of DNA repair genes in Arabidopsis mutants defective for two subunits specific to condensin II (Sakamoto et al. 2011). Moreover, smc41 nuclei display prominent RAD51 repair foci (Fig. 6E), as in atxr5/6 nuclei in which DNA damage is known to occur (Feng et al. 2017).

A flowering gene found by RNA-seq to be dramatically up-regulated in smc4-1 mutants is FLOWERING LOCUS $T(F T)$, which we verified using both gel-based RT-PCR assays (Fig. 6D) and qPCR (Fig. 7A). This likely explains the speedier transition to flowering observed under long-day conditions (16 h light, $8 \mathrm{~h}$ dark) for the original m73 mutant (genotype: smc4-1 CTD-OX cmt3) as well as the smc4-1 line resulting from repeated backcrossing to wild-type Col-0 (Fig. 7B,C).

Another important gene regulated by SMC4 is ROS1, which encodes a DNA glycosylase that facilitates the removal and replacement of methylated cytosines by DNA repair (Gong et al. 2002). ROS1 is up-regulated approximately fivefold in smc4-1 (Fig. 7D), indicating that condensin limits the basal expression level of ROS1. Consistent with previous studies (He et al. 2009; Lei et al. 2015), ROS1 expression is dependent on RdDM such that ROS1 expression is reduced in nrpd1 (pol IV) smc41 or nrpe1 (pol V) smc4-1 double mutants relative to the smc4-1 single mutant (Fig. 7D) yet still remains higher than in a pol $V$ single mutant. ROS 1 transcription levels positively correlate with RdDM-dependent methylation levels within a TE near the gene promoter (Lei et al. 2015; Williams et al. 2015). We observed a modest gain of methylation in all sequence contexts at this TE in the smc4-1 mutant (Supplemental Fig. S8). Condensin may thus limit the extent of RdDM at this site in wild-type plants.

\section{Discussion}

Previous case studies have documented roles for condensin in repressing specific loci. For instance, in budding yeast, condensin helps repress silent mating type loci and also represses Pol II transcription within the intergenic spacers of Pol I transcribed ribosomal RNA genes (Bhalla et al. 2002; Machin et al. 2004; He et al. 2016). Consistent with the latter studies, the non-SMC condensin subunit AtCAP-H2 of Arabidopsis localizes within the nucleolus (Fujimoto et al. 2005), and rRNA genes become decondensed in Arabidopsis RNAi lines with reduced SMC4 levels (Smith et al. 2014). In flies, condensin is involved in position effect variegation (Lupo et al. 2001; Dej et al. 2004). In C. elegans, a specialized condensin is involved in X-chromosome dosage control (Meyer 2010), and, in mice, condensin helps maintain T-cell quiescence (Rawlings et al. 2011). Our results extend these case 
A Chromosomal positions of SMC4-suppressed genes

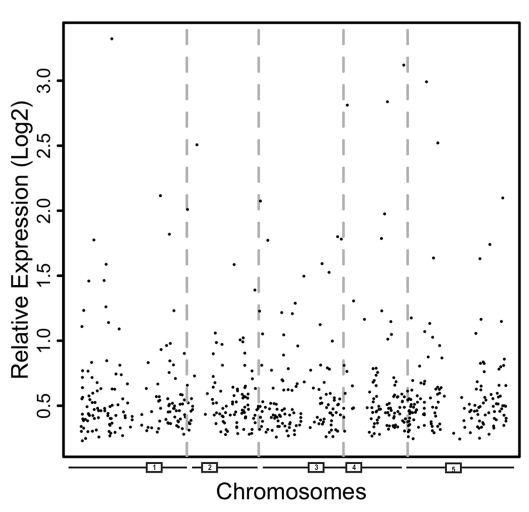

C Functional annotation of SMC4-suppressed genes

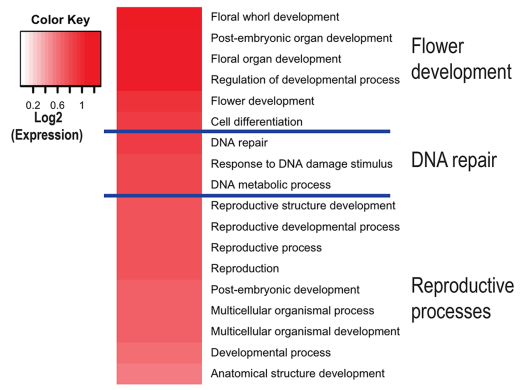

E RAD51 immunolocalization in leaf nuclei

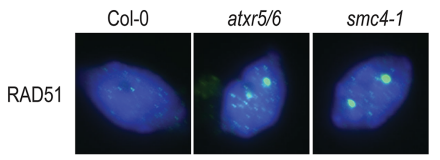

B DNA methylation of SMC4-suppressed genes

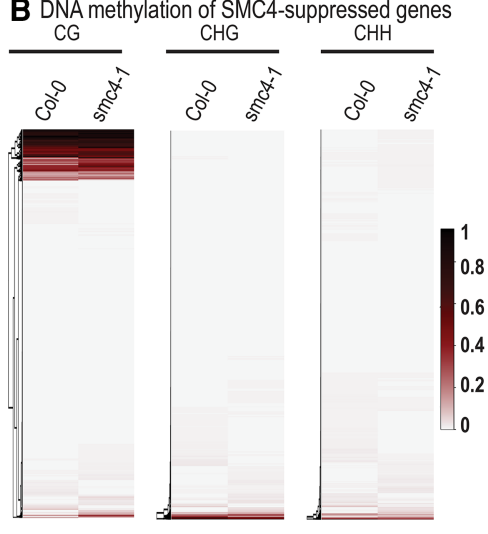

D SMC4-suppressed genes (RT-PCR)

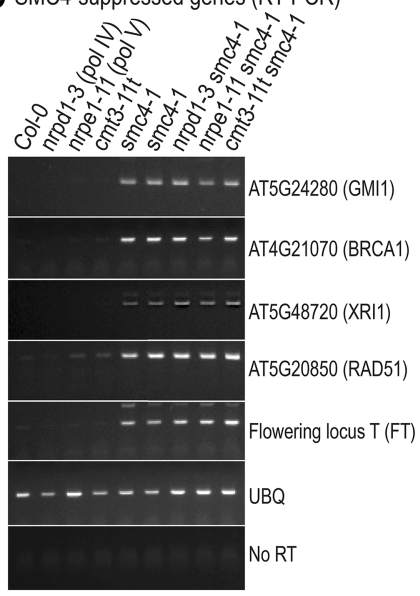

Figure 6. SMC4 represses protein-coding genes in addition to TEs. (A) Chromosomal positions and expression levels for protein-coding genes up-regulated in smc4-1. Estimated positions of centromeres are shown as boxes numbered accordingly for the five chromosomes. (B) Hierarchical clustering of the 533 SMC4-dependent protein-coding genes, displaying their relative DNA methylation levels on a scale of 0.0 (white) to 1.0 (red) in wild type and smc41 mutants. Columns represent data for each indicated genotype, and rows represent 200-bp windows covering the 533 genes. The rows were clustered by the complete agglomeration hierarchical clustering method, with Euclidean distance as a distance measure. $(C)$ Functional annotation of genes up-regulated in smc4-1. The color gradient reflects the degree of up-regulation in smc4-1 relative to wild type on a $\log _{2}$ scale. $(D)$ RT-PCR analysis of four DNA repair genes (GMI1, BRCA1, XRI1, and RAD51) and the flowering gene Flowering Locus $T(F T)$ predicted by RNA-seq to be up-regulated in smc4-1. UBQ and no reverse transcriptase (no RT) reactions served as controls. (E) smc4-1 nuclei display RAD51 enrichment foci indicative of DNA damage, consistent with the up-regulation of DNA repair genes. Images show immunolocalization of RAD51 in leaf nuclei counterstained with DAPI. The atxr5 atxr6 mutant, known to cause genome instability (Feng et al. 2017), served as a positive control for enhanced RAD51 foci. studies to a whole-genome level, made possible by the identification of smc4-1 as a viable, yet deleterious, mutation of the essential SMC4 gene. Our findings indicate that condensin acts as a corepressor of both genes and transposons, affecting hundreds of loci.

Our results indicate that condensin partners with symmetrical cytosine methylation and repressive histone modifications, particularly ATXR5/6-dependent $\mathrm{H} 3 \mathrm{~K} 27 \mathrm{me} 1$, in the repression of pericentromeric transposons. MET1 and ATXR5/6 are thought to be recruited to DNA replication forks by interacting with PCNA/Chuang et al. 1997; Raynaud et al. 2006; Hale et al. 2016). Interestingly, condensin has been detected at stalled DNA replication forks (D'Ambrosio et al. 2008) and shown to interact with DNA methyltransferases in mammalian cells (Geiman et al. 2004). These observations suggest that compact repressive chromatin structures might assemble quickly following DNA replication.

Condensin is needed for Drosophila Gypsy family retrotransposons to cluster within distinct chromatin bodies (Gerasimova et al. 2000) and for LTR retroelements in Schizosaccharomyces pombe to cluster in the vicinity of centromeres (Cam et al. 2008; Tanaka et al. 2012; Murton et al. 2016). How are these dispersed elements recognized and brought together? The fact that Arabidopsis loci that are heavily cytosine methylated or sparsely methylated can be regulated by condensin argues against DNA methylation as a primary determinant of condensin recruitment, as does the fact that flies and yeast do not appreciably methylate their DNA. Conserved histone modifications or histone variants present in all eukaryotes seem more likely as marks that enable condensin recruitment. Architectural chromatin proteins such as heterochromatin protein 1 (HP1) family members, Polycombrepressive complex 1 (PRC1) family proteins, or yeast silent information regulator (SIR) proteins might then serve as intermediaries for condensin recruitment (McBryant et al. 2006; Woodcock and Ghosh 2010; Grossniklaus and Paro 2014).

The mutation in the smc4-1 allele P22S is intriguing in that the substituted proline is highly conserved except in budding yeast, which has a serine at this position, as in smc4-1 (see Fig. 2D). It is plausible that the P-to-S mutation might be tolerated as a hypomorphic mutant in other model organisms, including mammals, in which null mutants are lethal. Given that somatic mutations in condensin subunits occur in multiple types of cancer (Leiserson et al. 2015; Uhlmann 2016), generating this mutation by gene editing might prove useful for genome-wide identification of loci that become derepressed in human cells when condensin function is compromised. 


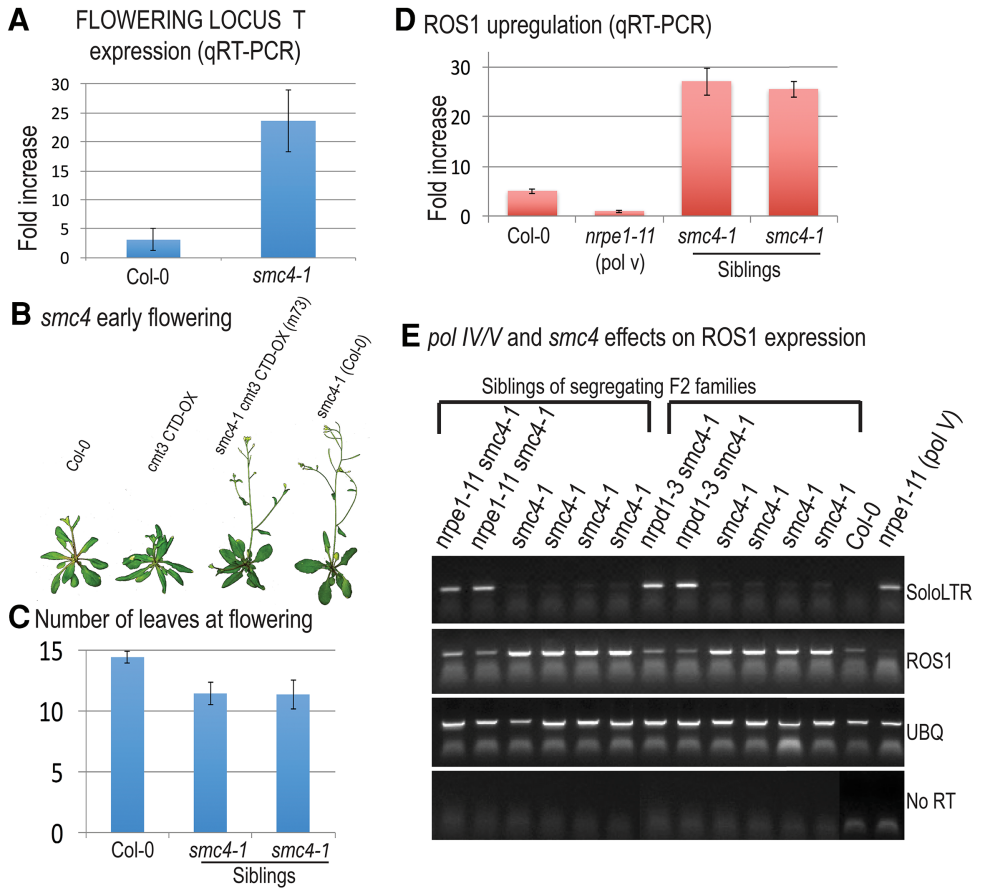

Figure 7. Early flowering and up-regulation of the ROS1 demethylase in smc4-1. (A) Early flowering of smc4-1 correlates with the up-regulation of $F T$ expression. The histogram shows qRT-PCR results for FT expression relative to ACTIN using RNA of pooled wildtype Col-0 or smc4-1 plants. Error bars indicate the standard deviation in three independent technical replicates. (B) smc4-1 mutants flower early. The images show 3-wk-old plants grown under long-day (16 h light) conditions, comparing wild type (Col-0), cmt3 CTD$O X, \mathrm{~m} 73$ (the smc4-1 mutation in the cmt3 CTD-OX background), and smc4-1 in an otherwise wild-type Col-0 background. (C) Quantification of the number of vegetative leaves present at the time of flowering. The histograms show mean values and standard deviations (error bars) determined for 18 Col-0, 19 smc4-1 (Col-0), or 18 smc4-1 (Col-0) plants. The two sets of smc4-1 plants represent independent families derived from F2 siblings resulting from the second round of Col-0 X smc4-1 backcrossing. (D) qRT-PCR analysis of ROS1 expression in wild type, nrpe1-11 (pol V), and smc4-1 mutants. The two smc4-1 samples represent siblings of the F2 family resulting from the second round of Col-0 X smc4-1 backcrossing. The data were normalized to ACTIN, with error bars indicating standard deviations based on three independent technical replicates. (E) RT-PCR analysis of ROS1 gene expression in smc4-1, nrpd1-3 (pol IV) smc4-1, and nrpe1-11 (pol V) smc4-1 mutants. SoloLTR served as a control for the Pol IV- and Pol V-dependent $\mathrm{RdDM}$ effect. $U B Q$ and no reverse transcriptase (no RT) reactions served as controls.

\section{Materials and methods}

Plant materials

A. thaliana ecotype Col-0 was used in all experiments. met1-3 and ddm1-2 were described in Saze et al. (2003), and nrpd1-3 (pol IV) and nrpe1-11 (pol V) mutants were described previously (Onodera et al. 2005; Pontes et al. 2006). atxr5 atxr6 was provided by Scott Michaels, and met1-1 was provided by Eric Richards. drm2-2 and $c m t 3-11 t$ were obtained from the Arabidopsis Biological Resource Center (ABRC). The cmt3-11t met1-1 double mutant was created by crossing $\mathrm{cmt3}-11 \mathrm{t}$ to met1-1 (Pontvianne et al. 2013). The SMC4 T-DNA insertion mutant line SAIL-86-D02 (Siddiqui et al. 2006) was obtained from the ABRC. Mutant lines of the condensin subunits cap-e1 (CS84719), cap-d2 (SALK_077796C), cap-d3 (SALK_094776), cap-g2 (SALK-049790C), and cap-h2 (SALK_059304) were obtained from the ABRC.

The CTD-OX transgene corresponding to NRPE1 sequences encoding amino acids 1249-1976 was cloned into pEarleyGate 202 (Earley et al. 2006) and then transformed into cmt3-11t by the floral dip method (Clough and Bent 1998). Transgenic plants were selected by Basta herbicide resistance, and 431 positive transformants were further screened to identify 242 lines with Basta resistance, segregating 3:1. These 242 lines were next subjected to Southern blot analysis to identify plants with single T-DNA left and right border fragments. Line \#389 (cmt3 OX-CTD) was chosen for EMS mutagenesis. An $O X-$ CTD line was obtained by backcrossing \#389 (cmt3 OX-CTD) to Col-0 and identifying F2 individuals lacking cmt3 mutant alleles.

\section{EMS mutagenesis and genetic mapping}

Approximately 22,000 $\mathrm{cm} t 3 C T D$-OX line seeds were mutagenized with EMS as described (Kim et al. 2006), except that the EMS concentration was $0.3 \%$. Seeds of resulting M1 plants were harvested, and resulting M2 plants with a wild-type (as opposed to SDC) phenotype were identified. Bulked-segregant analysis and sequence-based mapping were conducted as described by Hartwig et al. (2012). Briefly, putative suppressor mutants were first backcrossed to the $\mathrm{cm}$ t3 CTD-OX parental line, and resulting F1 plants were grown and allowed to self-pollinate to produce F2 seeds. F2 plants were then grown, and 50 plants displaying SDC or wild-type phenotypes were pooled, and their DNA was subjected to library construction and deep sequencing, with an estimated genome coverage of $30 \times$. The cmt3 CTD-OX line was also sequenced. Candidate EMS-induced mutations absent in the parental line and displaying high allele frequencies in suppressor mutants were confirmed by sequencing of PCR-amplified genomic DNA.

\section{Complementation of smc4-1 by an SMC4 transgene}

Plant transformation vector pHPT carrying a full-length wildtype SMC4 genomic clone insert (Siddiqui et al. 2006) was provided by Daniel Riggs. The plasmid was transformed into Agrobacterium tumefaciens strain GV3101 and subsequently used to transform smc4-1 mutant plants. Transformants were initially selected by hygromycin resistance and verified using PCR assays.

\section{DNA genotyping analyses}

For T-DNA mutant genotyping, genomic DNA from 2-wk-old plants was isolated using CTAB extraction. GoTaq Green master mix (2×; Promega) was mixed with 100 ng of genomic DNA and appropriate primer pairs. PCR products were resolved and visualized by agarose gel electrophoresis. Genotyping primers are listed in Supplemental Table S4. Genotyping of smc4-1 involved PCR amplification followed by HindIII digestion; the smc4-1 mutant was cleaved, whereas the wild-type gene was not. 
DNA methylation analyses

Genomic DNA was isolated from 2-wk-old plants using the Nucleon PhytoPure DNA extraction kit (Amersham). Chop-PCR assays were performed using $100 \mathrm{ng}$ of restriction endonucleasedigested ("chopped") genomic DNA as in Blevins et al. (2017). Bisulfite sequencing analysis of the ROS1 promoter regions was according to Blevins et al. (2014). In brief, PCR fragments amplified from bisulfite-treated DNA were cloned into pGEM-T-Easy and sequenced using a T7 primer. Forty-eight sequences per amplicon were analyzed in CyMATE (Hetzl et al. 2007). ChopPCR and bisulfite sequencing primers are listed in Supplemental Table S4.

\section{Semiquantitative $R T-P C R$}

Total RNA was extracted from three to four leaves of 2-wk-old plants using the Spectrum plant total RNA kit (Sigma-Aldrich). RNA $(1.5 \mu \mathrm{g})$ was then treated using a Turbo DNA-free kit (Thermo Fisher Scientific) and used for random-primed cDNA synthesis using SuperScript III reverse transcriptase (Invitrogen). Resulting cDNA was used for PCR amplification using GoTaq Green polymerase (Promega) and primers listed in Supplemental Table S4.

RNA-seq and data analysis

Total RNA was extracted from 2-wk-old Arabidopsis leaves using TRI reagent (MRC, Inc.). Libraries for three biological replicates of each genotype were constructed and sequenced using the Illumina NextSeq 500 platform. TrueSeq adaptor sequences were trimmed using Trimmomatic version 0.33 . Reads post-trimming were filtered with a quality score cutoff of 20 and length cutoff of 30 . Filtered reads were mapped to the TAIR10 genome using TopHat 2.0.10. The number of uniquely mapped reads corresponding to annotated protein-coding genes or TEs were computed using HTSeq-count version 0.5.4p1 (Anders et al. 2015). The DESeq2 package in $\mathrm{R}$ (Love et al. 2014) was used for calling significantly differentially expressed genes and TEs. Differentially expressed genes were defined as those with $<5 \%$ FDR and a $P$-value cutoff of 0.01 , whereas derepressed TEs were defined as those with a $<5 \%$ FDR and a fold change in log base 2 of $>2$. The identification and evaluation of statistical significance for intersections among multiple sets of derepressed TEs were conducted using the "SuperExactTest" package in R (Wang et al. 2015).

Whole-genome bisulfite sequencing and data analysis

Genomic DNA was isolated from 2-wk-old Arabidopsis leaves using an Illustra Nucleon PhytoPure DNA extraction kit (GE Healthcare). Three biological replicates of each genotype were submitted to the Beijing Genome Institute (http://www. genomics.cn/en) for bisulfite treatment, library preparation, and Illumina sequencing. For data analysis, adapters were trimmed, low-quality sequences $(q<20)$ were filtered, and clean reads were mapped to the TAIR10 genome using Bismark (Krueger and Andrews 2011). Methylated cytosines supported by at least five reads were passed to the MethylKit package in $\mathrm{R}$ for further analysis (Akalin et al. 2012). For calling differentially methylated cytosines in CG, $\mathrm{CHG}$, and $\mathrm{CHH}$ contexts, threshold differences of at least $50 \%, 25 \%$, and $15 \%$ methylation, respectively, were required along with $P$-values of $<0.01$. Differentially methylated regions were defined as regions containing at least 10 differentially methylated cytosines in each 200-base-pair (bp) sliding window, with a step size of $150 \mathrm{bp}$. DNA methylation levels were compared between each wild-type and mutant window using Fisher's exact test with a $P$-value cutoff of 0.05 . The $P$-values were adjusted using the standard Benjamini-Hochberg method to control for FDRs.

Small RNA-seq and blot analyses

Total RNA was extracted from 2-wk-old Arabidopsis seedlings using TRIzol (ThermoFisher Scientific) and submitted to Fasteris SA (http://www.fasteris.com) for library construction and small RNA-seq, performed on an Illumina HiSeq 2000 platform. RNA size fractionation was conducted as in Blevins et al. (2014) except that gel slices containing RNAs of 15-30 nt were used rather than RNAs of 15-45 nt. For small RNAs, reads were first adaptortrimmed to remove TruSeq $3^{\prime}$ small RNA adaptor sequences using Trimmomatic version 0.33 (Bolger et al. 2014) and then quality-trimmed, setting the cutoff threshold for average base quality score at 20 over a window of 3 bases. Reads $>15$ bases post-trimming were excluded. Trimmed and filtered reads were then mapped to the TAIR 10 reference genome sequence using Bowtie version 1.1.2 (Langmead et al. 2009), and only perfectly matched 21- to 25-nt RNAs were analyzed further. Any reads aligning to 45S rRNA, chloroplast, or mitochondria were excluded. Read alignments were further filtered for size classes 21 and $24 \mathrm{nt}$.

For small RNA Northern blot analyses, $\sim 100 \mu \mathrm{g}$ of total RNA was extracted from 2-wk-old seedlings using TRIzol (ThermoFisher Scientific) and then size-fractionated on RNeasy minicolumns (Qiagen) (Blevins et al. 2006). The low-molecular-weight RNA fraction was then used as described previously (Blevins et al. 2015).

Accession numbers

Sequence data generated in this study have been deposited in the NCBI Sequence Read Archive (http://www.ncbi.nlm.nih.gov/sra) under accession number SRP105760.

\section{Acknowledgments}

We thank Dan Riggs for providing the SMC4 genomic clone, James Ford and the Indiana University (IU) Center for Genomics and Bioinformatics, and Jim Powers and the IU Light Microscopy Imaging Center. This work was supported by funds made available to C.S.P. by National Institutes of Health grant GM077590 and Gordon and Betty Moore Foundation grant 3036 and funds made available to C.S.P. as an Investigator of the Howard Hughes Medical Institute. T.B. and E.H.T. generated the cmt3 CTD-OX line that provided the basis for the genetic screen, based on initial experiments by J.R.H., who engineered the CTD-OX transgene and identified the dominant-negative nrpe1 phenotype that it induces. J.W. and T.B. developed the bioinformatics pipeline for mapping EMS-generated point mutations. J.W. and R.P. performed all bioinformatics except those for Figure 4F and Supplemental Figure S5, conducted by F.W. J.W. conducted all remaining experiments. J.W. and C.S.P. wrote the manuscript.

\section{References}

Akalin A, Kormaksson M, Li S, Garrett-Bakelman FE, Figueroa ME, Melnick A, Mason CE. 2012. methylKit: a comprehensive R package for the analysis of genome-wide DNA methylation profiles. Genome Biol 13: R87.

Allen E, Xie Z, Gustafson AM, Carrington JC. 2005. microRNAdirected phasing during trans-acting siRNA biogenesis in plants. Cell 121: 207-221. 
Anders S, Pyl PT, Huber W. 2015. HTSeq-a Python framework to work with high-throughput sequencing data. Bioinformatics 31: 166-169.

Bhalla N, Biggins S, Murray AW. 2002. Mutation of YCS4, a budding yeast condensin subunit, affects mitotic and nonmitotic chromosome behavior. Mol Biol Cell 13: 632-645.

Blevins T, Rajeswaran R, Shivaprasad PV, Beknazariants D, SiAmmour A, Park HS, Vazquez F, Robertson D, Meins F Jr, Hohn T, et al. 2006. Four plant Dicers mediate viral small RNA biogenesis and DNA virus induced silencing. Nucleic Acids Res 34: 6233-6246.

Blevins T, Pontvianne F, Cocklin R, Podicheti R, Chandrasekhara C, Yerneni S, Braun C, Lee B, Rusch D, Mockaitis K, et al. 2014. A two-step process for epigenetic inheritance in Arabidopsis. Mol Cell 54: 30-42.

Blevins T, Podicheti R, Mishra V, Marasco M, Wang J, Rusch D, Tang H, Pikaard CS. 2015. Identification of Pol IV and RDR2-dependent precursors of $24 \mathrm{nt}$ siRNAs guiding de novo DNA methylation in Arabidopsis. Elife 4: e09591.

Blevins T, Wang J, Pflieger D, Pontvianne F, Pikaard CS. 2017. Hybrid incompatibility caused by an epiallele. Proc Natl Acad Sci 114: 3702-3707.

Bolger AM, Lohse M, Usadel B. 2014. Trimmomatic: a flexible trimmer for Illumina sequence data. Bioinformatics 30: 2114-2120.

Bostick M, Kim JK, Esteve PO, Clark A, Pradhan S, Jacobsen SE. 2007. UHRF1 plays a role in maintaining DNA methylation in mammalian cells. Science 317: 1760-1764.

Brzeski J, Jerzmanowski A. 2003. Deficient in DNA methylation 1 (DDM1) defines a novel family of chromatin-remodeling factors. J Biol Chem 278: 823-828.

Cam HP, Noma K, Ebina H, Levin HL, Grewal SI. 2008. Host genome surveillance for retrotransposons by transposon-derived proteins. Nature 451: 431-436.

Cao X, Jacobsen SE. 2002. Role of the Arabidopsis DRM methyltransferases in de novo DNA methylation and gene silencing. Curr Biol 12: 1138-1144.

Cao X, Aufsatz W, Zilberman D, Mette MF, Huang MS, Matzke M, Jacobsen SE. 2003. Role of the DRM and CMT3 methyltransferases in RNA-directed DNA methylation. Curr Biol 13: $2212-2217$.

Chuang LS, Ian HI, Koh TW, Ng HH, Xu G, Li BF. 1997. Human DNA-(cytosine-5) methyltransferase-PCNA complex as a target for p21WAF1. Science 277: 1996-2000.

Clough SJ, Bent AF. 1998. Floral dip: a simplified method for Agrobacterium-mediated transformation of Arabidopsis thaliana. Plant J 16: 735-743.

D'Ambrosio C, Schmidt CK, Katou Y, Kelly G, Itoh T, Shirahige K, Uhlmann F. 2008. Identification of cis-acting sites for condensin loading onto budding yeast chromosomes. Genes DeV 22: 2215-2227.

Dej KJ, Ahn C, Orr-Weaver TL. 2004. Mutations in the Drosophila condensin subunit dCAP-G: defining the role of condensin for chromosome condensation in mitosis and gene expression in interphase. Genetics 168: 895-906.

Earley KW, Haag JR, Pontes O, Opper K, Juehne T, Song K, Pikaard CS. 2006. Gateway-compatible vectors for plant functional genomics and proteomics. Plant J 45: 616-629.

Feng W, Hale CJ, Over RS, Cokus SJ, Jacobsen SE, Michaels SD. 2017. Large-scale heterochromatin remodeling linked to overreplication-associated DNA damage. Proc Natl Acad Sci 114: 406-411.

Fransz P, De Jong JH, Lysak M, Castiglione MR, Schubert I. 2002. Interphase chromosomes in Arabidopsis are organized as well defined chromocenters from which euchromatin loops emanate. Proc Natl Acad Sci 99: 14584-14589.

Fujimoto S, Yonemura M, Matsunaga S, Nakagawa T, Uchiyama S, Fukui K. 2005. Characterization and dynamic analysis of Arabidopsis condensin subunits, AtCAP-H and AtCAP-H2. Planta 222: 293-300.

Geiman TM, Sankpal UT, Robertson AK, Chen Y, Mazumdar M, Heale JT, Schmiesing JA, Kim W, Yokomori K, Zhao Y, et al. 2004. Isolation and characterization of a novel DNA methyltransferase complex linking DNMT3B with components of the mitotic chromosome condensation machinery. Nucleic Acids Res 32: 2716-2729.

Gerasimova TI, Byrd K, Corces VG. 2000. A chromatin insulator determines the nuclear localization of DNA. Mol Cell 6: $1025-1035$.

Gong Z, Morales-Ruiz T, Ariza RR, Roldan-Arjona T, David L, Zhu JK. 2002. ROS1, a repressor of transcriptional gene silencing in Arabidopsis, encodes a DNA glycosylase/lyase. Cell 111: 803-814.

Grossniklaus U, Paro R. 2014. Transcriptional silencing by polycomb-group proteins. Cold Spring Harb Perspect Biol 6: a019331.

Haag JR, Pikaard CS. 2011. Multisubunit RNA polymerases IV and V: purveyors of non-coding RNA for plant gene silencing. Nat Rev Mol Cell Biol 12: 483-492.

Hale CJ, Potok ME, Lopez J, Do T, Liu A, Gallego-Bartolome J, Michaels SD, Jacobsen SE. 2016. Identification of multiple proteins coupling transcriptional gene silencing to genome stability in Arabidopsis thaliana. PLoS Genet 12: e1006092.

Hartwig B, James GV, Konrad K, Schneeberger K, Turck F. 2012. Fast isogenic mapping-by-sequencing of ethyl methanesulfonate-induced mutant bulks. Plant Physiol 160: 591-600.

Hashimoto $\mathrm{H}$, Horton JR, Zhang X, Bostick M, Jacobsen SE, Cheng X. 2008. The SRA domain of UHRF1 flips 5-methylcytosine out of the DNA helix. Nature 455: 826-829.

He XJ, Hsu YF, Zhu S, Liu HL, Pontes O, Zhu J, Cui X, Wang CS, $\mathrm{Zhu}$ JK. 2009. A conserved transcriptional regulator is required for RNA-directed DNA methylation and plant development. Genes Dev 23: 2717-2722.

He HI, Zhang S, Wang D, Hochwagen A, Li F. 2016. Condensin promotes position effects within tandem DNA repeats via the RITS complex. Cell Rep 14: 1018-1024.

Henderson IR, Jacobsen SE. 2008. Tandem repeats upstream of the Arabidopsis endogene SDC recruit non-CG DNA methylation and initiate siRNA spreading. Genes Dev 22: 1597-1606.

Hetzl J, Foerster AM, Raidl G, Mittelsten Scheid O. 2007. CyMATE: a new tool for methylation analysis of plant genomic DNA after bisulphite sequencing. Plant J 51: 526-536.

Hirano T. 2016. Condensin-based chromosome organization from bacteria to vertebrates. Cell 164: 847-857.

Iyer LM, Anantharaman V, Wolf MY, Aravind L. 2008. Comparative genomics of transcription factors and chromatin proteins in parasitic protists and other eukaryotes. Int I Parasitol 38: $1-31$.

Jacob Y, Martienssen R. 2012. All packed up and ready to go. Science 336: 1391-1392.

Jacob Y, Feng S, LeBlanc CA, Bernatavichute YV, Stroud H, Cokus S, Johnson LM, Pellegrini M, Jacobsen SE, Michaels SD. 2009. ATXR5 and ATXR6 are H3K27 monomethyltransferases required for chromatin structure and gene silencing. Nat Struct Mol Biol 16: 763-768.

Jacob Y, Stroud H, Leblanc C, Feng S, Zhuo L, Caro E, Hassel C, Gutierrez C, Michaels SD, Jacobsen SE. 2010. Regulation of 
heterochromatic DNA replication by histone H3 lysine 27 methyltransferases. Nature 466: 987-991.

Jeddeloh JA, Stokes TL, Richards EJ. 1999. Maintenance of genomic methylation requires a SWI2/SNF2-like protein. Nat Genet 22: 94-97.

Jeppsson K, Kanno T, Shirahige K, Sjogren C. 2014. The maintenance of chromosome structure: positioning and functioning of SMC complexes. Nat Rev Mol Cell Biol 15: 601-614.

Kim Y, Schumaker KS, Zhu JK. 2006. EMS mutagenesis of Arabidopsis. Methods Mol Biol 323: 101-103.

Krueger F, Andrews SR. 2011. Bismark: a flexible aligner and methylation caller for Bisulfite-Seq applications. Bioinformatics 27: 1571-1572.

Langmead B, Trapnell C, Pop M, Salzberg SL. 2009. Ultrafast and memory-efficient alignment of short DNA sequences to the human genome. Genome Biol 10: R25.

Law JA, Jacobsen SE. 2010. Establishing, maintaining and modifying DNA methylation patterns in plants and animals. Nat Rev Genet 11: 204-220.

Lei M, Zhang H, Julian R, Tang K, Xie S, Zhu JK. 2015. Regulatory link between DNA methylation and active demethylation in Arabidopsis. Proc Natl Acad Sci 112: 3553-3557.

Leiserson MD, Vandin F, Wu HT, Dobson JR, Eldridge JV, Thomas JL, Papoutsaki A, Kim Y, Niu B, McLellan M, et al. 2015. Pan-cancer network analysis identifies combinations of rare somatic mutations across pathways and protein complexes. Nat Genet 47: 106-114.

Lippman Z, Gendrel AV, Black M, Vaughn MW, Dedhia N, McCombie WR, Lavine K, Mittal V, May B, Kasschau KD, et al. 2004. Role of transposable elements in heterochromatin and epigenetic control. Nature 430: 471-476.

Lorkovic ZJ, Naumann U, Matzke AJ, Matzke M. 2012. Involvement of a GHKL ATPase in RNA-directed DNA methylation in Arabidopsis thaliana. Curr Biol 22: 933-938.

Love MI, Huber W, Anders S. 2014. Moderated estimation of fold change and dispersion for RNA-seq data with DESeq2. Genome Biol 15: 550.

Lupo R, Breiling A, Bianchi ME, Orlando V. 2001. Drosophila chromosome condensation proteins Topoisomerase II and Barren colocalize with Polycomb and maintain Fab-7 PRE silencing. Mol Cell 7: 127-136.

Machin F, Paschos K, Jarmuz A, Torres-Rosell J, Pade C, Aragon L. 2004. Condensin regulates rDNA silencing by modulating nucleolar Sir2p. Curr Biol 14: 125-130.

Mallory A, Vaucheret H. 2010. Form, function, and regulation of ARGONAUTE proteins. Plant Cell 22: 3879-3889.

Matzke MA, Mosher RA. 2014. RNA-directed DNA methylation: an epigenetic pathway of increasing complexity. Nat Rev Genet 15: 394-408.

McBryant SJ, Adams VH, Hansen JC. 2006. Chromatin architectural proteins. Chromosome Res 14: 39-51.

Meyer BJ. 2010. Targeting X chromosomes for repression. Curr Opin Genet Dev 20: 179-189.

Moissiard G, Cokus SI, Cary J, Feng S, Billi AC, Stroud H, Husmann D, Zhan Y, Lajoie BR, McCord RP, et al. 2012. MORC family ATPases required for heterochromatin condensation and gene silencing. Science 336: 1448-1451.

Mourrain P, Beclin C, Elmayan T, Feuerbach F, Godon C, Morel JB, Jouette D, Lacombe AM, Nikic S, Picault N, et al. 2000. Arabidopsis SGS2 and SGS3 genes are required for posttranscriptional gene silencing and natural virus resistance. Cell 101: $533-542$.

Murton HE, Grady PJ, Chan TH, Cam HP, Whitehall SK. 2016. Restriction of retrotransposon mobilization in Schizosacchar- omyces pombe by transcriptional silencing and higher-order chromatin organization. Genetics 203: 1669-1678.

Onodera Y, Haag JR, Ream T, Costa Nunes P, Pontes O, Pikaard CS. 2005. Plant nuclear RNA polymerase IV mediates siRNA and DNA methylation-dependent heterochromatin formation. Cell 120: 613-622.

Peragine A, Yoshikawa M, Wu G, Albrecht HL, Poethig RS. 2004. SGS3 and SGS2/SDE1/RDR6 are required for juvenile development and the production of trans-acting siRNAs in Arabidopsis. Genes Dev 18: 2368-2379.

Pontes O, Li CF, Costa Nunes P, Haag J, Ream T, Vitins A, Jacobsen SE, Pikaard CS. 2006. The Arabidopsis chromatin-modifying nuclear siRNA pathway involves a nucleolar RNA processing center. Cell 126: 79-92.

Pontvianne F, Blevins T, Chandrasekhara C, Mozgova I, Hassel C, Pontes OM, Tucker S, Mokros P, Muchova V, Fajkus J, et al. 2013. Subnuclear partitioning of rRNA genes between the nucleolus and nucleoplasm reflects alternative epiallelic states. Genes Dev 27: 1545-1550.

Rawlings JS, Gatzka M, Thomas PG, Ihle JN. 2011. Chromatin condensation via the condensin II complex is required for peripheral T-cell quiescence. EMBO J 30: 263-276.

Raynaud C, Sozzani R, Glab N, Domenichini S, Perennes C, Cella R, Kondorosi E, Bergounioux C. 2006. Two cell-cycle regulated SET-domain proteins interact with proliferating cell nuclear antigen (PCNA) in Arabidopsis. Plant J 47: 395-407.

Sakamoto T, Inui YT, Uraguchi S, Yoshizumi T, Matsunaga S, Mastui M, Umeda M, Fukui K, Fujiwara T. 2011. Condensin II alleviates DNA damage and is essential for tolerance of boron overload stress in Arabidopsis. Plant Cell 23: 3533-3546.

Saze H, Mittelsten Scheid O, Paszkowski J. 2003. Maintenance of CpG methylation is essential for epigenetic inheritance during plant gametogenesis. Nat Genet 34: 65-69.

Siddiqui NU, Rusyniak S, Hasenkampf CA, Riggs CD. 2006. Disruption of the Arabidopsis SMC4 gene, AtCAP-C, compromises gametogenesis and embryogenesis. Planta 223: 990-997.

Simon L, Voisin M, Tatout C, Probst AV. 2015. Structure and function of centromeric and pericentromeric heterochromatin in Arabidopsis thaliana. Front Plant Sci 6: 1049.

Smith SJ, Osman K, Franklin FC. 2014. The condensin complexes play distinct roles to ensure normal chromosome morphogenesis during meiotic division in Arabidopsis. Plant $I$ 80: 255-268.

Soppe WI, Jasencakova Z, Houben A, Kakutani T, Meister A, Huang MS, Jacobsen SE, Schubert I, Fransz PF. 2002. DNA methylation controls histone $\mathrm{H} 3$ lysine 9 methylation and heterochromatin assembly in Arabidopsis. EMBO $I$ 21: 6549-6559.

Stroud H, Hale CJ, Feng S, Caro E, Jacob Y, Michaels SD, Jacobsen SE. 2012. DNA methyltransferases are required to induce heterochromatic re-replication in Arabidopsis. PLoS Genet 8: e1002808.

Stroud H, Greenberg MV, Feng S, Bernatavichute YV, Jacobsen SE. 2013. Comprehensive analysis of silencing mutants reveals complex regulation of the Arabidopsis methylome. Cell 152: 352-364.

Stroud H, Do T, Du J, Zhong X, Feng S, Johnson L, Patel DI, Jacobsen SE. 2014. Non-CG methylation patterns shape the epigenetic landscape in Arabidopsis. Nat Struct Mol Biol 21: $64-72$.

Tanaka A, Tanizawa H, Sriswasdi S, Iwasaki O, Chatterjee AG, Speicher DW, Levin HL, Noguchi E, Noma K. 2012. Epigenetic regulation of condensin-mediated genome organization during the cell cycle and upon DNA damage through histone H3 lysine 56 acetylation. Mol Cell 48: 532-546. 
Wang et al.

Uhlmann F. 2016. SMC complexes: from DNA to chromosomes. Nat Rev Mol Cell Biol 17: 399-412.

Vazquez F, Vaucheret H, Rajagopalan R, Lepers C, Gasciolli V, Mallory AC, Hilbert JL, Bartel DP, Crete P. 2004. Endogenous trans-acting siRNAs regulate the accumulation of Arabidopsis mRNAs. Mol Cell 16: 69-79.

Wang MH, Zhao YZ, Zhang B. 2015. Efficient test and visualization of multi-set intersections. Sci Rep 5: 16923.

Wendte JM, Pikaard CS. 2017. The RNAs of RNA-directed DNA methylation. Biochim Biophys Acta 1860: 140-148.

Williams BP, Pignatta D, Henikoff S, Gehring M. 2015. Methylation-sensitive expression of a DNA demethylase gene serves as an epigenetic rheostat. PLoS Genet 11: e1005142.

Woo HR, Pontes O, Pikaard CS, Richards EJ. 2007. VIM1, a methylcytosine-binding protein required for centromeric heterochromatinization. Genes Dev 21: 267-277.
Wood AJ, Severson AF, Meyer BJ. 2010. Condensin and cohesin complexity: the expanding repertoire of functions. Nat Rev Genet 11: 391-404.

Woodcock CL, Ghosh RP. 2010. Chromatin higher-order structure and dynamics. Cold Spring Harb Perspect Biol 2: a000596.

Zemach A, Kim MY, Hsieh PH, Coleman-Derr D, EshedWilliams L, Thao K, Harmer SL, Zilberman D. 2013. The Arabidopsis nucleosome remodeler DDM1 allows DNA methyltransferases to access $\mathrm{Hl}$-containing heterochromatin. Cell 153: 193-205.

Zhang X, Yazaki J, Sundaresan A, Cokus S, Chan SW, Chen H, Henderson IR, Shinn P, Pellegrini M, Jacobsen SE, et al. 2006. Genome-wide high-resolution mapping and functional analysis of DNA methylation in Arabidopsis. Cell 126: 1189-1201. Zhou M, Law JA. 2015. RNA Pol IV and V in gene silencing: rebel polymerases evolving away from Pol II's rules. Curr Opin Plant Biol 27: 154-164. 


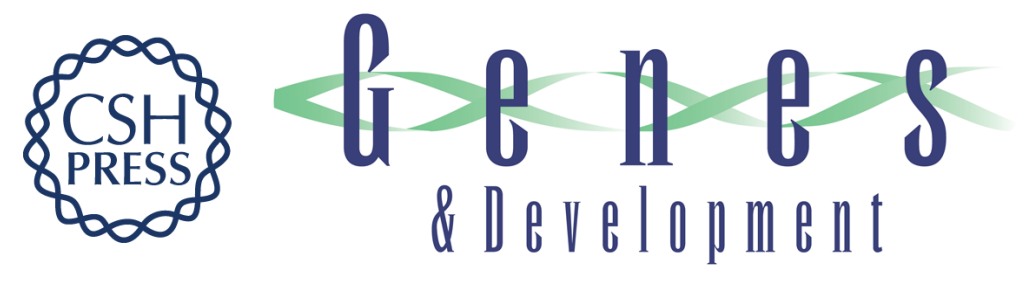

\section{Mutation of Arabidopsis SMC4 identifies condensin as a corepressor of pericentromeric transposons and conditionally expressed genes}

Jing Wang, Todd Blevins, Ram Podicheti, et al.

Genes Dev. 2017, 31: originally published online September 7, 2017

Access the most recent version at doi:10.1101/gad.301499.117

\section{Supplemental http://genesdev.cshlp.org/content/suppl/2017/09/07/gad.301499.117.DC1 Material}

References This article cites 86 articles, 25 of which can be accessed free at: http://genesdev.cshlp.org/content/31/15/1601.full.html\#ref-list-1

Creative This article is distributed exclusively by Cold Spring Harbor Laboratory Press for the first Commons six months after the full-issue publication date (see

License http://genesdev.cshlp.org/site/misc/terms.xhtml). After six months, it is available under a Creative Commons License (Attribution-NonCommercial 4.0 International), as described at http://creativecommons.org/licenses/by-nc/4.0/.

Email Alerting Receive free email alerts when new articles cite this article - sign up in the box at the top Service right corner of the article or click here.

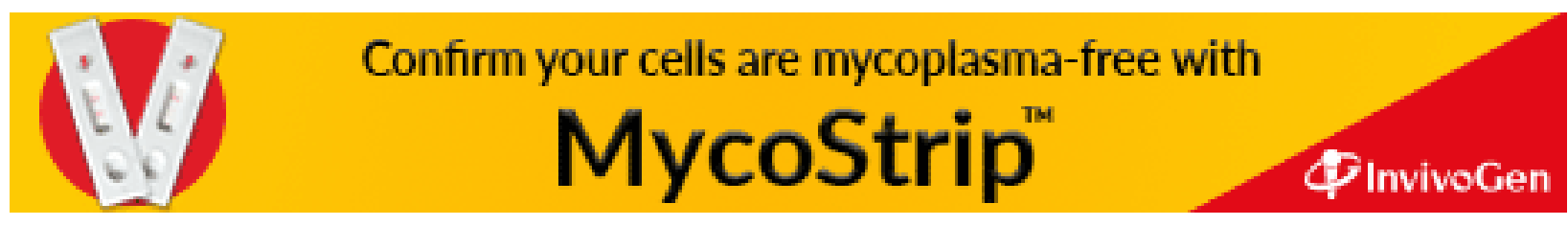

